\title{
Generation of optical frequency combs in fibres
}

M. Zajnulina, J. M. Chavez Boggio, A. A. Rieznik, R. Haynes, M. M. Roth

M. Zajnulina, J. M. Chavez Boggio, A. A. Rieznik, R. Haynes, M. M. Roth, "Generation of optical frequency combs in fibres," Proc. SPIE 8775, Microstructured and Specialty Optical Fibres II, 87750C (3 May 2013); doi: $10.1117 / 12.2017167$

SPIE. Event: SPIE Optics + Optoelectronics, 2013, Prague, Czech Republic 


\title{
Generation of Optical Frequency Combs in Fibres
}

\author{
M. Zajnulina ${ }^{1}$, J.M. Chavez Boggio ${ }^{1}$, A.A. Rieznik ${ }^{2}$, R. Haynes ${ }^{1}$, M.M. Roth ${ }^{1}$ \\ ${ }^{1}$ innoFSPEC-VKS, Leibniz-Institut für Astrophysik, An der Sternwarte 16, D-14482 Potsdam, \\ Germany \\ ${ }^{2}$ Instituto Tecnologico de Buenos Aires and CONICET, Buenos Aires, Argentina
}

\begin{abstract}
We numerically investigated the possibility of generating high-quality ultra-short optical pulses with broad frequencycombs spectra in a system consisting of three optical fibres. In this system, the first fibre is a conventional single-mode fibre, the second one is erbium-doped, and the last one is a low-dispersion fibre. The system is pumped with a modulated sine-wave generated by two equally intense lasers with the wavelengths $\lambda_{1}$ and $\lambda_{2}$ such that their central wavelength is at $\lambda_{c}=\left(\lambda_{1}+\lambda_{2}\right) / 2=1531 \mathrm{~nm}$. The modelling was performed using the generalised nonlinear Schrödinger equation which includes the Kerr and Raman effects, as well as the higher-order dispersion and gain. We took a close look at the pulse evolution in the first two stages and studied the pulse behaviour depending on the group-velocity dispersion and the nonlinear parameter of first fibre, as well as the initial laser frequency separation. For these parameters, the optimum lengths of fibre 1 and 2 were found that provide low-noise pulses. To characterise the pulse energy content, we introduced a figure of merit that was dependent on the group-velocity dispersion, the nonlinearity of fibre 1, and the laser separation.
\end{abstract}

Keywords: Optical frequency combs, Nonlinear Schrödinger equation, Four-wave mixing, Ultra-short pulses, Pulse compression, Astronomy, Astrocombs

\section{INTRODUCTION}

Most spectrographs for ground-based or space telescopes are calibrated with the help of spectral lamps (cf. [1]). The disadvantage of this calibration method is that the spectra of such lamps provide non-uniformly spaced emission lines with unequal intensities. Moreover, the lamps tend to a quick aging which has a frequency drift as a result. Thus, alternative light sources with well-defined emission lines are highly required for the calibration purposes. An ideal highprecision calibrator should provide uniformly spaced and equally intense spectral lines over several hundreds of nanometres. It was shown that the use of optical frequency combs generated in passive mode-locked lasers fulfilled well the requirements for a high-precision calibration [2-9]. However, mode-locked lasers can be complex and costly, their extensive deployment in the telescopes around the world therefore difficult. In this paper, we propose a simple, low-cost, and stable all-fibre scheme for generation of optical frequency combs (OFCs). In particular, we optimise the system parameters so that it allows generation of low-noise optical pulses within the broad frequency-comb spectra. In section 2 of this paper, we introduce the experimental setup for generation of OFCs and the according model based on the generalised nonlinear Schrödinger equation which we solve numerically in order to obtain optimised parameters of the system. In section 3, we present our results and, finally draw a conclusion in section 4.

\section{SETUP AND THEORETICAL BACKGROUND}

\subsection{Experimental setup}

In Fig. 1, one can see an experimental setup that allows to generate broad and stable optical frequency combs. It consists of three coupled fibres being pumped with two coupled equally intense continuous wave (CW) lasers. One of the lasers has a fixed wavelength, the other one is tunable so that the resulting modulated sine-wave has the central wavelength is

Micro-structured and Specialty Optical Fibres II, edited by Kyriacos Kalli,

Jiri Kanka, Alexis Mendez, Proc. of SPIE Vol. 8775, 87750C · (C) 2013 SPIE

CCC code: $0277-786 \mathrm{X} / 13 / \$ 18 \cdot$ doi: $10.1117 / 12.2017167$ 
at $1531 \mathrm{~nm}$. The first fibre of the system is a conventional single-mode fibre, the second one is erbium-doped, whereas the last one is a low-dispersion fibre [10].

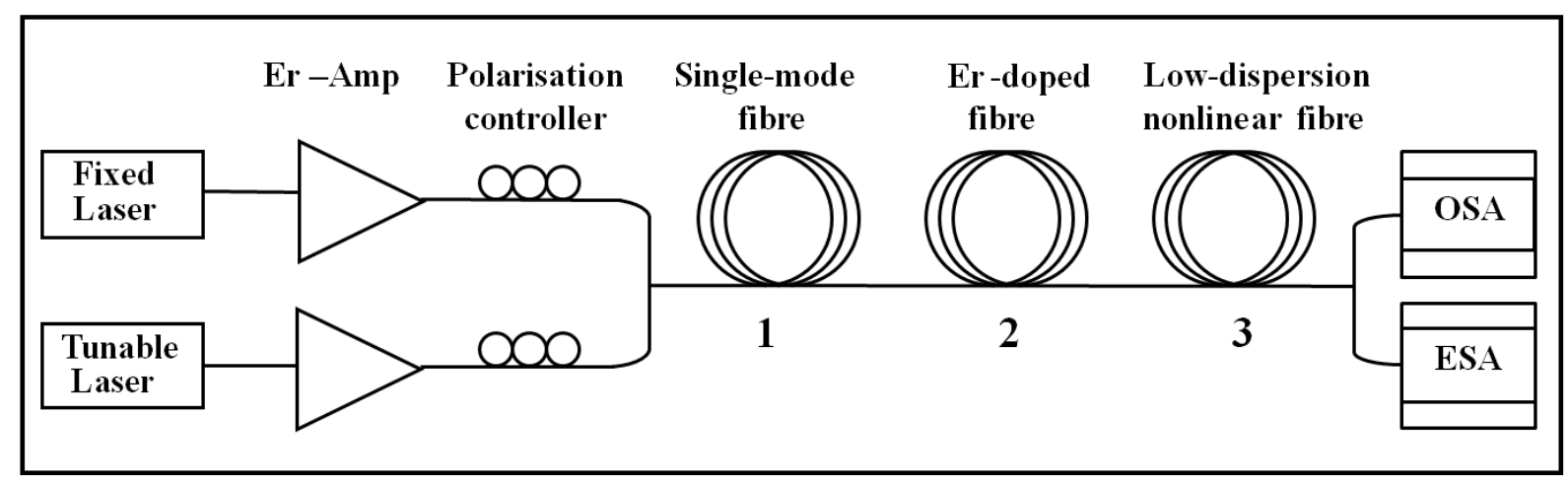

Fig. 1: Experimental setup. OSA: Optical spectrum analyser, ESA: electrical spectrum analyser

After the initial modulated sine-wave enters the first fibre, its dynamics are governed by the optical properties of silica. As the wave propagates through the fibre, its spectrum initially containing only two lines begins to gain additional lines due to the four-wave mixing process (FWM). The new lines are equidistant, their frequency spacing coincides with the initial laser separation: a frequency comb arises. Due to the joint action of the cross-phase modulation effect (XPM) and the negative group-velocity dispersion (GVD), every hump of the sine-wave begins to be compressed into a soliton. The process of the pulse compression is finished after a certain propagation length along which the counterbalancing of the XPM and the GVD is reached. The soliton consists now of an intense narrow spike positioned against the background of a broad pedestal [13]. As a result of the compression process, a train of optical solitons accompanied by the pedestal arises. The pulse repetition rate of corresponds to the value of the initial laser separation: $v_{\text {repeat }}=L S$.

After the propagation through the first fibre, the intensities of the pulses reach the values of a few tens of Watts. In the second Er-doped fibre, the lower-frequency components of the solitons' spectra experience the Raman gain at the expense of the higher-frequency components $[14,15]$. The cross-phase modulation effect leads to the appearance of additional spectral lines and so to the broadening of the frequency comb. Again, the spectrally broadened pulses undergo strong compression due to the interplay between the XPM and the anomalous GVD. The intensities of the pulses can increase to a few $\mathrm{kW}$ as they propagated through the second fibre, whereas the pulses are compressed to the femtosecond range. After these pulses enter the third fibre, their spectra are subjected to both, the cross-phase modulation and the Raman effects which lead to a further broadening of the frequency comb. Since the dispersion of the third fibre is kept low, no pulse compression occurs in the third fibre. Not only the GVD, but also higher-order dispersion, especially the third-order dispersion (TOD), play an important role in silica. Normally, the contribution of the TOD is negligible compared to the GVD. However, a considerable amount of asymmetrical broadening in the time domain will be produced by the TOD for ultra-short pulses, i.e. pulses that arise and propagate in the second and the third fibre [15]. Moreover, the TOD causes a slight increase in the pedestal of the compressed pulses and a decrease of their peak intensity [16].

\subsection{Model}

To model the experimental setup we use the generalised non-linear Schrödinger equation (GNLS) [10, 17]:

$$
\frac{\partial A}{\partial z}=i \sum_{k=2}^{N} \frac{i^{k}}{k !} \beta_{k} \frac{\partial^{k} A}{\partial t^{k}}+i \gamma\left(1+\frac{i}{\omega_{0}} \frac{\partial}{\partial t}\right)\left(A \int_{-\infty}^{\infty} R\left(t^{\prime}\right)\left|A\left(t-t^{\prime}\right)\right|^{2} d t^{\prime}\right)+\left(g-\frac{\alpha}{2}\right) A,
$$

where $A=A(z, t)$ denotes the complex envelope of the electric field obtained via the slowly varying envelope approximation, $\beta_{k}=\left(\frac{\partial^{k} \beta}{\partial \omega^{k}}\right)_{\omega=\omega_{0}}$ represents the value of the dispersion order at the carrier frequency $\omega_{0}$, and $\gamma$ is the 
nonlinear coefficient defined as $\gamma=\frac{\omega_{0} n_{2}}{c \cdot S}$ with $n_{2}$ being the nonlinear refractive index of silica and $S$ the effective mode area. For the dispersion order, we use $N=10$ for the first fibre and $N=3$ for both, the second and the third fibre. The delayed Raman response is included into the GNLS via the response function

$R(t)=\left(1-f_{R}\right) \delta(t)+f_{R} h_{R}(t)$, where $f_{R}$ is the fractional contribution of the Raman response and $h_{R}$ is defined as follows:

$$
\begin{array}{r}
h_{R}(t)=\left(f_{a}+f_{c}\right) h_{a}(t)+f_{b} h_{b}(t), \\
h_{a}(t)=\frac{\tau_{1}^{2}+\tau_{2}^{2}}{\tau_{1} \tau_{2}^{2}} \exp \left(-\frac{t}{\tau_{2}}\right) \sin \left(\frac{t}{\tau_{1}}\right), \\
h_{b}(t)=\left(\frac{2 \tau_{b}-t}{\tau_{b}^{2}}\right) \exp \left(-\frac{t}{\tau_{b}}\right)
\end{array}
$$

with $\tau_{1}$ and $\tau_{2}$ being the characteristic times of the Raman response with the typical values for silica $\tau_{1}=12.2 f s$ and $\tau_{2}=32 \mathrm{fs}$. All other parameters have the following values: $\tau_{b}=96 \mathrm{fs}, f_{a}=0.75, f_{b}=0.21$, and $f_{R}=0.245$. The last term on the right-hand side of Eq. (1) represents the frequency dependent loss $(\alpha)$ due to the absorption by Erbium and the correspondent Erbium-gain $(g)$ based on the amplified spontaneous emission. Both, $g$ and $\alpha$ are valid for second fibre and are 0 for the first and the third fibre. The initial condition for Eq. (1) is given by:

$$
A_{0}(z=0, t)=\sqrt{P_{0}} \sin \left(\omega_{c} t\right)+\sqrt{n_{0 / \text { rand }}(t)} \exp ^{i \varphi_{\text {rand }}(t)},
$$

where the first term mimics the radiation of two lasers with the frequencies $\omega_{1}$ and $\omega_{2}$ being equally positioned around the central frequency $\omega_{c}$ which corresponds to the central wavelength at $1531 \mathrm{~nm} . P_{0}$ denotes the input power of both lasers. The second term of Eq. (3) describes the noise contribution with the maximal noise power $n_{0}$ and the noise phase $\varphi$, which are both randomly distributed over time.

The numerical solution of Eqs. (1), (2), and (3) was performed using the interaction picture method in combination with the local error method. We used 216 sample points and a 256 ps temporal window.

\section{RESULTS}

\subsection{Optimum lengths}

After the optical solitons are formed in the first fibre from the initial sine-wave, they undergo periodic modulation as well as temporal pulse compression as they propagate further through the fibre. We define the optimum length of the fibre as a length at which the pulses reached their first highest peak during the evolution, which also corresponds to the first minimum of the pulse width $[10,11,12]$. The same procedure is applied to the second fibre while the length of the third fibre is kept constant and has the value of $L_{3 F}=1.27 \mathrm{~m}$ during the whole set of simulations. The choice of the optimum lengths for the first and the second fibre allows us not only an effective minimisation of the fibre material which is preferable from the point of view of the costs savings, but also provides us with high-quality pulses, since the impact of the higher-order dispersion is negligible for comparably short optimum lengths. Specifically for the second fibre, the noise evolution due to the noise amplification is kept low.

In the following example, we vary the group-velocity dispersion $\beta_{2 / 1 F}$ and the nonlinear parameter $\gamma_{1 F}$ of the first fibre, as well as the initial laser frequency separation $L S$ and look for optimum lengths of fibre 1 and 2 . The following parameters of the second fibre (indexed by $2 F$ ) are kept fixed as well as the parameters of the third one (indexed by $3 F$ ): $\beta_{2 / 2 F}=-14 \mathrm{ps}^{2} / \mathrm{km}, \beta_{3 / 2 F}=0.1 \mathrm{ps}^{3} / \mathrm{km}, \gamma_{2 F}=2.5(\mathrm{~W} \cdot \mathrm{km})^{-1}, \beta_{2 / 3 F}=0.05 \mathrm{ps}^{2} / \mathrm{km}$,

$\beta_{3 / 3 F}=0.0081 \mathrm{ps}^{3} / \mathrm{km}$, and $\gamma_{3 F}=10(\mathrm{~W} \cdot \mathrm{km})^{-1}$. The maximum of the initial noise power was chosen to be $n_{0 / \text { rand }}=2 P_{0} e^{-10}$, where $P_{0}$ is the initial peak power of the input wave. 
Fig. 2 shows the optimum lengths of the first (Fig. 2(A)) and the second (Fig. 2 (B)) fibre depending on the initial input power $P_{0}$ and three different values of the GVD, i.e $\beta_{2 / 1 F}=-7.5 \mathrm{ps}^{2} / \mathrm{km},-15 \mathrm{ps}^{2} / \mathrm{km}$, and $-30 \mathrm{ps}^{2} / \mathrm{km}$. The nonlinear parameter is chosen to be $\gamma_{1 F}=2(\mathrm{~W} \cdot \mathrm{km})^{-1}$ and the laser separation $L S=0.08 \mathrm{THz}$. As one can see in Fig. 2 (A), there is a plateau at low input powers, where the optimum length of the first fibre is constant for each specific value of the GVD. The input-power region of this plateau increases with the absolute value of the GVD. The reason for this is that the high anomalous group-velocity dispersion prohibits the formation of optical solitons out of the initial sinewave because it counteracts the nonlinear effects. Only if the input power is high enough to evoke nonlinear effects such that they are able to counterbalance the GVD, is the formation of a train of optical solitons possible. From this threshold of the input power, the optimum length starts decreasing with the value of $P_{0}$. In general, one can effectively decrease the optimum length of the first fibre by choosing a fibre with high value of anomalous group-velocity dispersion. However, if one takes a look into Fig. 2 (B), one realises that a high value of the anomalous GVD of the first fibre yields a longer optimum length of the second fibre. Obviously, the optimum length of fibre 2 increases with the value of the anomalous group-velocity dispersion of fibre 1 , whereas it decreases with the input power.
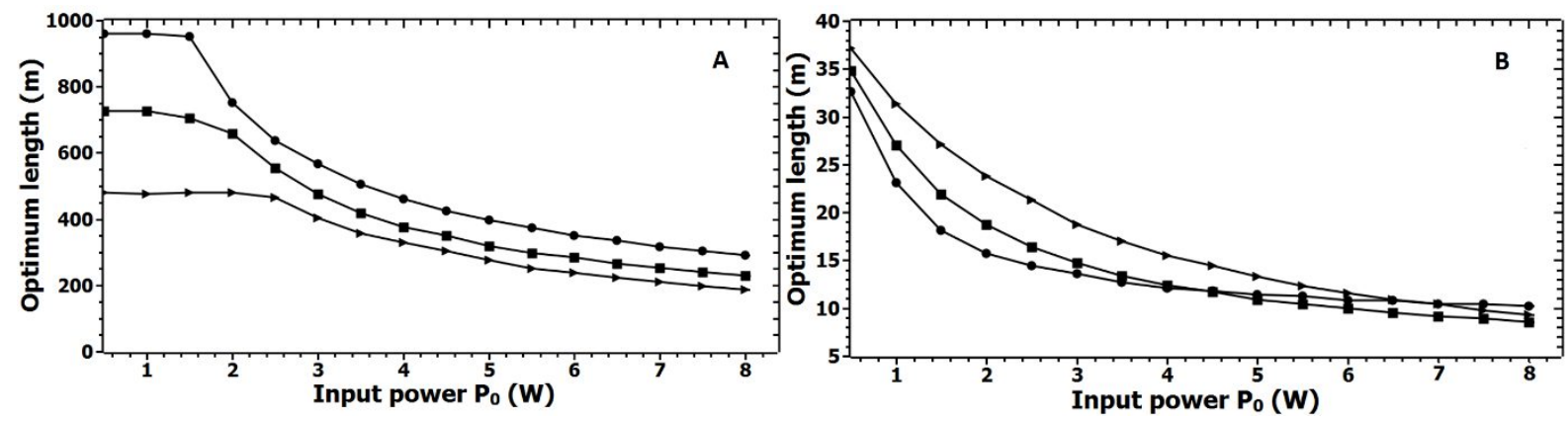

Fig. 2: Optimum lengths of the 1. fibre (A) and the 2. fibre (B) depending on the input power $P_{0}$ and different values of

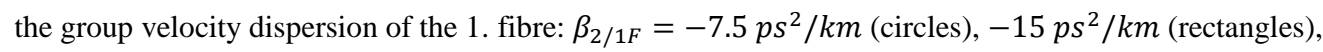
and $-30 p s^{2} / k m$ (triangles)

As for the intensity noise, the first fibre makes only a small contribution to its evolution: for the chosen value of the initial maximum noise power, i.e. for $n_{0 / \text { rand }}=2 P_{0} e^{-10}$, the intensity noise lies below $0.6 \%$ for all considered values of the GVD. In the second fibre, the intensity noise reaches up to $80 \%$ at low powers, but rapidly decreases as the input power increases and goes down to maximal $0.8 \%$ for $P_{0} \geq 3 \mathrm{~W}$. Thus, for an effective saving of the fibre material, one should choose a system with a fibre having a GVD of $\beta_{2 / 1 F}=-15 \mathrm{ps}^{2} / \mathrm{km}$ for the first stage and, to minimise the noise evolution, the input powers greater than $3 \mathrm{~W}$. For the further studies, we will use $\beta_{2 / 1 F}=-15 \mathrm{ps}^{2} / \mathrm{km}$ as the value of the GVD of the first fibre.

Now we consider the dependence of the optimum lengths from three different values of the nonlinear parameter of the first fibre: $\gamma_{1 F}=2(W \cdot \mathrm{km})^{-1}, 6(W \cdot \mathrm{km})^{-1}$, and $10(W \cdot \mathrm{km})^{-1}$. The initial laser separation is the same as previously. As one can see in Fig. 3, the optimum lengths of the first fibre decrease as the nonlinear parameter and the input power increase. For $\gamma_{1 F}=6(\mathrm{~W} \cdot \mathrm{km})^{-1}$ and $10(\mathrm{~W} \cdot \mathrm{km})^{-1}$, the first fibre has no plateaus with constant values of the optimum lengths for low input powers (Fig. 3 (A)). This is because the nonlinearities are high enough to induce generation of optical solitons even at low powers. However, such solitons are not stable as they propagate through the second fibre (Fig. 3 (B)): they tend to break having soliton fission as a result as the input power increases. This is clearly seen at the example with $\gamma_{1 F}=10(\mathrm{~W} \cdot \mathrm{km})^{-1}$ when the value of the optimum length drops dramatically at $P_{0}=5 \mathrm{~W}$. As for the case with $\gamma_{1 F}=6(\mathrm{~W} \cdot \mathrm{km})^{-1}$, the soliton fission will occur at $P_{0}=9 \mathrm{~W}$, which is not included into the graph, but was observed in our simulations. 

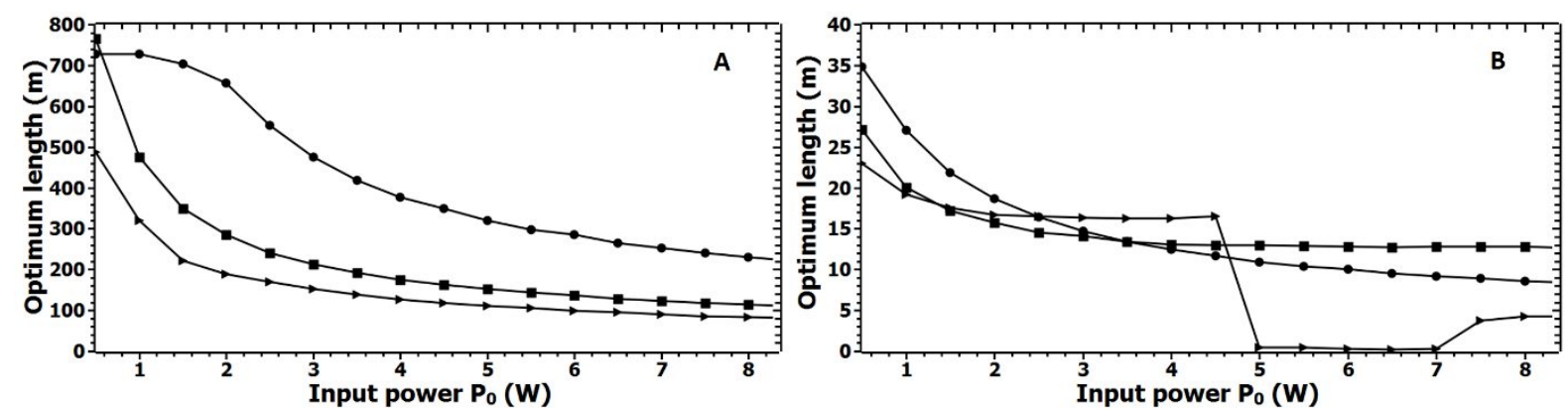

Fig. 3: Optimum lengths of the 1. fibre (A) and the 2. fibre (B) depending on the input power $P_{0}$ and different values of the nonlinear parameter of the 1. fibre: $\gamma_{1 F}=2(\mathrm{~W} \cdot \mathrm{km})^{-1}$ (circles), $6(\mathrm{~W} \cdot \mathrm{km})^{-1}$ (rectangles), and $10(W \cdot k m)^{-1}$ (triangles)

The intensity noise of the system directly depends on the nonlinear parameter: the higher the value of $\gamma_{1 F}$, the more the system is subjected to noise evolution. Already in the first fibre, starting with the value of ca. $0.2 \%$ at $P_{0}=0.5 \mathrm{~W}$ for all chosen nonlinear parameters, the intensity noise rapidly increases with the input power if the nonlinearity of the fibre is high. The following values of the noise are achieved at $P_{0}=8.0 \mathrm{~W}: 1 \%$ for $\gamma_{1 F}=6(\mathrm{~W} \cdot \mathrm{km})^{-1}$ and ca. $8 \%$ for $\gamma_{1 F}=$ $10(W \cdot \mathrm{km})^{-1}$, whereas for $\gamma_{1 F}=2(W \cdot \mathrm{km})^{-1}$ the intensity noise increases only to $0.6 \%$. Also in the second fibre, the intensity noise increases with the value of the nonlinear parameter and the input power. At $P_{0}=8.0 \mathrm{~W}$ we have $0.8 \%$ for $\gamma_{1 F}=2(\mathrm{~W} \cdot \mathrm{km})^{-1}, 1.2 \%$ for $\gamma_{1 F}=6(\mathrm{~W} \cdot \mathrm{km})^{-1}$, and $10 \%$ for $\gamma_{1 F}=10(\mathrm{~W} \cdot \mathrm{km})^{-1}$. Therefore, in order to minimise the noise evolution, it is preferable to choose fibres with low nonlinearities for the first stage. We will continue our studies when the nonlinear coefficient of the first fibre is $2(\mathrm{~W} \cdot \mathrm{km})^{-1}$.

Having $\beta_{2 / 1 F}=-15 p s^{2} / \mathrm{km}$ and $\gamma_{1 F}=2(\mathrm{~W} \cdot \mathrm{km})^{-1}$, we now check the dependence of the optimum lengths on three different value of the initial laser frequency separation: $L S=0.04 \mathrm{THz}, 0.08 \mathrm{THz}$, and $0.16 \mathrm{THz}$. As one can in Fig. 4 (A), the optimum length of the first fibre decreases as the laser separation and the input power increase. If the pulses follow each other at high repetition rate, which is the case for high values of the laser separation, their phases have a stronger interaction with each other having a broadening of the single pulse spectra as a result. In this case, the anomalous group-velocity dispersion has a bigger impact on the pulse evolution meaning that higher intensities and so higher nonlinearities are needed to counterbalance it. Thus, the region of the input power values at which the optimum length has a plateau extends to the higher values of $P_{0}$ for higher values of the laser separation. As for the second fibre (Fig. 4 (B)), the optimum lengths decrease as the input power increases and the laser separation decreases. Since amplifying fibres are expensive compared to conventional single-mode fibres, it is preferable to choose an input wave with a low value of the laser separation to minimise the costs. To avoid plateaus in the first fibre, one should use input powers greater than $2 \mathrm{~W}$.
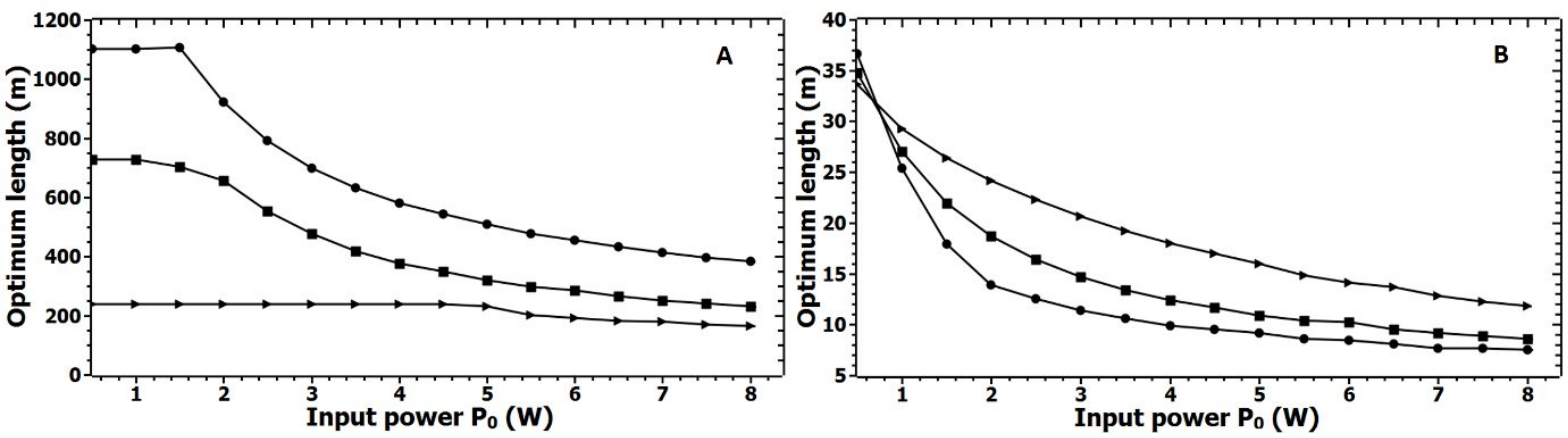

Fig. 4: Optimum lengths of the 1. fibre (A) and the 2. fibre (B) depending on the input power $P_{0}$ and different values of the input laser separation: $L S=0.04 \mathrm{THz}$ (circles), $0.08 \mathrm{THz}$ (rectangles), and $0.16 \mathrm{THz}$ (triangles) 
The laser separation has hardly an impact on the evolution of the intensity noise in the first and second fibre. For all chosen values of the initial laser separation and the input power, the intensity noise remains below $1 \%$ in both fibres.

As we have seen, the technique of the fibre optimisation via the choice of the optimum lengths allows low-noise generation and propagation of optical solitons. The amplifying character of the second fibre has only a weak contribution to the noise evolution in that case. However, the high nonlinearity of the third fibre, i.e $\gamma_{3 F}=10(\mathrm{~W} \cdot \mathrm{km})^{-1}$, induces an increase of the system noise generally by the factor of 3 . Thus, the quality of the broad frequency comb generated by the third fibre is degraded as a result of the higher levels of noise. Choosing a fibre with a lower nonlinearity for the third stage will result in the generation of a comb with a less broadened spectrum. Therefore, depending on the system requirements, one will need to optimise the nonlinearity of the third fibre, in order to obtain a broadest possible spectrum with a low level of noise.

\subsection{Figure of merit}

Now we check the effectiveness of the systems in terms of the energy content. We define the figure of merit (FoM) as the ratio between the peak power $P_{\text {peak }}$ and the averaged power $\bar{P}$ of single optical pulses:

$$
F o M=\frac{P_{\text {peak }}}{\bar{P}} \text {. }
$$

The figure of merit not only provides us with information how much energy is saved within a pulse, but also gives an indirect clue about the effectiveness of the pulse compression: a higher value of the peak power and so of the figure of merit coincides with a lower value of the pulse width.

We start this study when the group-velocity dispersion is chosen to be $\beta_{2 / 1 F}=-7.5 \mathrm{ps}^{2} / \mathrm{km},-15 \mathrm{ps}^{2} / \mathrm{km}$, and $-30 \mathrm{ps}^{2} / \mathrm{km}$ again, whereas the nonlinear parameter has the value of $\gamma_{1 F}=2(\mathrm{~W} \cdot \mathrm{km})^{-1}$ and the laser separation is $L S=0.08 \mathrm{THz}$. As one can see in Fig. 5 (A), the figure of merit of the first fibre increases as the absolute value of GVD and the input power increase. The curves flatten towards the higher value of the input power denoting that less energy goes into the optical pulses and more energy is stored in the surrounding wings (pedestal) of the pulses. In general, higher values of the input power induce the growth of a higher pedestal as the pulses evolve out of the initial sine-wave inside the first fibre. As for the second fibre (Fig. 5 (B)), the figure of merit increases, reaches a maximum and then decreases as the input power increases. The position of the maximum depends on the value of the GVD: the lower the absolute value of the GVD, the less input power is needed to reach the maximum of the figure of merit in second fibre. It is important that the values of the curve maxima do not depend on the specific value of $\beta_{2 / 1 F}$. The decrease of the FoM after its maximum arises again due to the evolution of higher pedestals as the input power increases. Due to the smooth increase and slow decrease of the curve, we choose $\beta_{2 / 1 F}=-15 \mathrm{ps}^{2} / \mathrm{km}$ for our further studies.
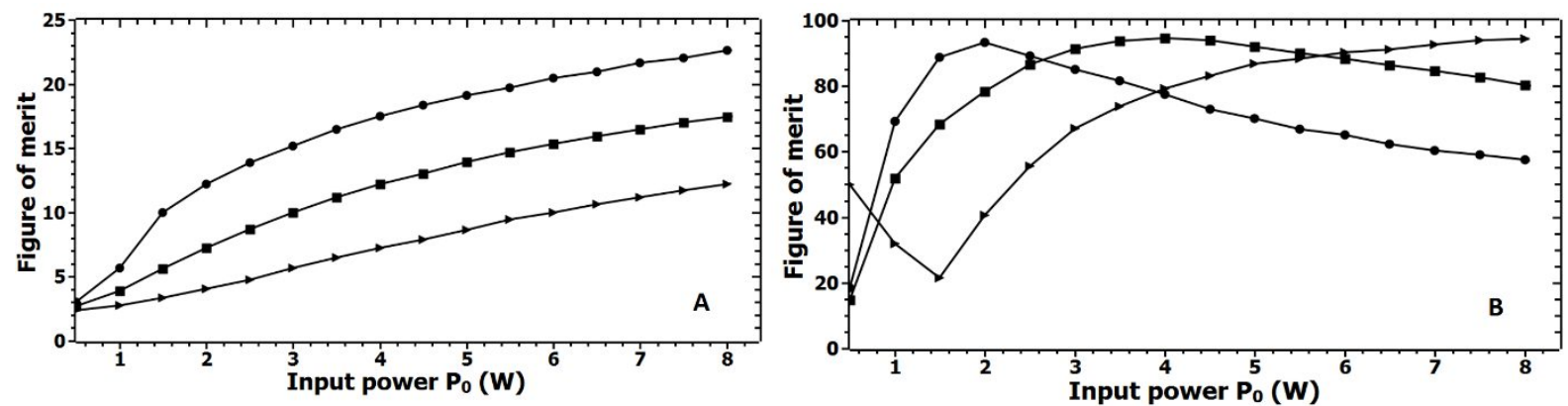

Fig. 5: Figure of merit of the 1. fibre (A) and the 2. fibre (B) depending on the input power $P_{0}$ and different values of

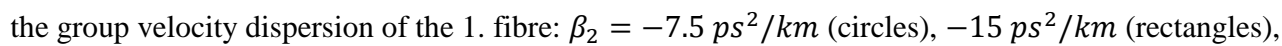
and $-30 p s^{2} / k m$ (triangles) 
Next we vary the nonlinear parameter of the first fibre: $\gamma_{1 F}=2(W \cdot \mathrm{km})^{-1}, 6(\mathrm{~W} \cdot \mathrm{km})^{-1}$, and $10(\mathrm{~W} \cdot \mathrm{km})^{-1}$. The initial laser frequency separation remains the same, i.e. $L S=0.08 \mathrm{THz}$. The figure of merit of the first fibre (Fig. 6 (A)) increases as the nonlinear parameter and the input power increase. As for the second fibre (Fig. 6 (B)), the curves increase, reach a maximum, and decrease as the input power grows. The negative slope of each single curve after the maximum depends on the value of the nonlinear parameter: the higher the value of $\gamma_{1 F}$, the faster the curve decreases. The maxima themselves have equal values of the FoM and shift towards the lower powers as the nonlinear parameter increases. The local maxima at $P_{0}=4 \mathrm{~W}$ and $P_{0}=7 \mathrm{~W}$ occur or slightly precede the input power values at which the soliton fission takes place. Due to the smooth increase and a slow decrease of the figure-of-merit values in the second fibre, it is preferable to choose a fibre with a low nonlinearity for the first stage. We now continue our investigations with $\gamma_{1 F}=2(W \cdot \mathrm{km})^{-1}$.
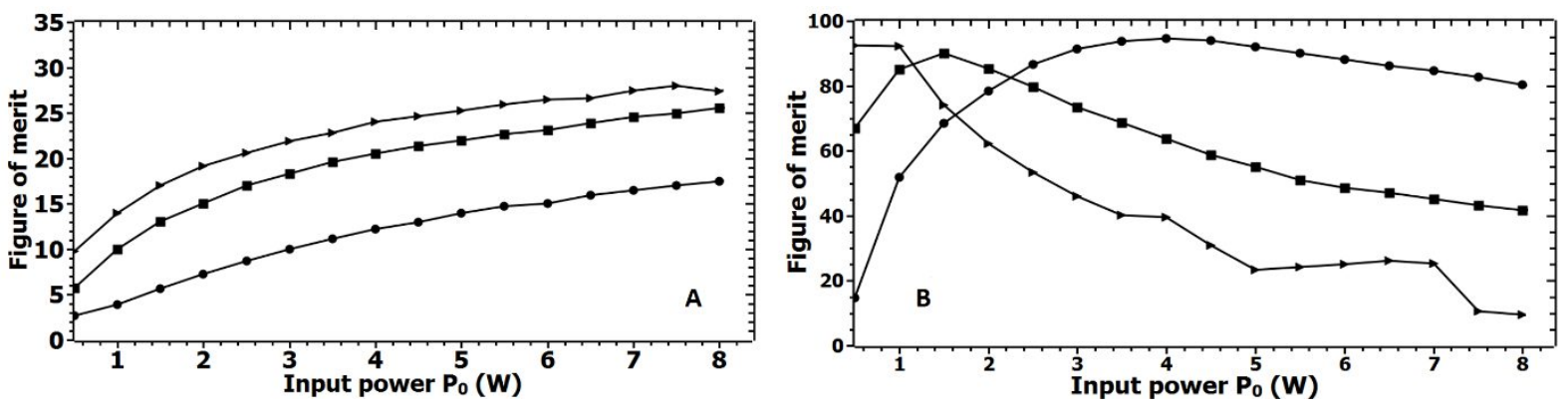

Fig. 6: Figure of merit of the 1. fibre (A) and the 2. fibre (B) depending on the input power $P_{0}$ and different values of the nonlinear parameter of the 1. fibre: $\gamma=2(W \cdot \mathrm{km})^{-1}$ (circles), $6(W \cdot \mathrm{km})^{-1}$ (rectangles), and $10(W \cdot k m)^{-1}$ (triangles)

Now, having $\beta_{2 / 1 F}=-15 \mathrm{ps}^{2} / \mathrm{km}$ and $\gamma_{1 F}=2(\mathrm{~W} \cdot \mathrm{km})^{-1}$, we check the dependence of the figure of merit on the initial laser frequency separation. Again, we choose $L S=0.04 \mathrm{THz}, 0.08 \mathrm{THz}$, and $0.16 \mathrm{THz}$. As presented in Fig. 7 (A), the figure of merit of the first fibre increases with the input power and decreases with the value of the laser separation. If the pulses rapidly follow each other in the time domain, which is the case when the value of the laser separation is high, there is a stronger interaction of the pulse pedestals increasing the general pedestal background, which then decreases the figure of merit of the second fibre (Fig. 7 (B)) as the laser separation increases. For specific values of $L S=0.04 \mathrm{THz}$ and $L S=0.08 \mathrm{THz}$ the curves start decreasing after they reached a maximum which occurs due to the evolution of higher pulse pedestals as the input power grows. Obviously, the optimum behaviour of the system is provided when $L S=0.08 \mathrm{THz}$.
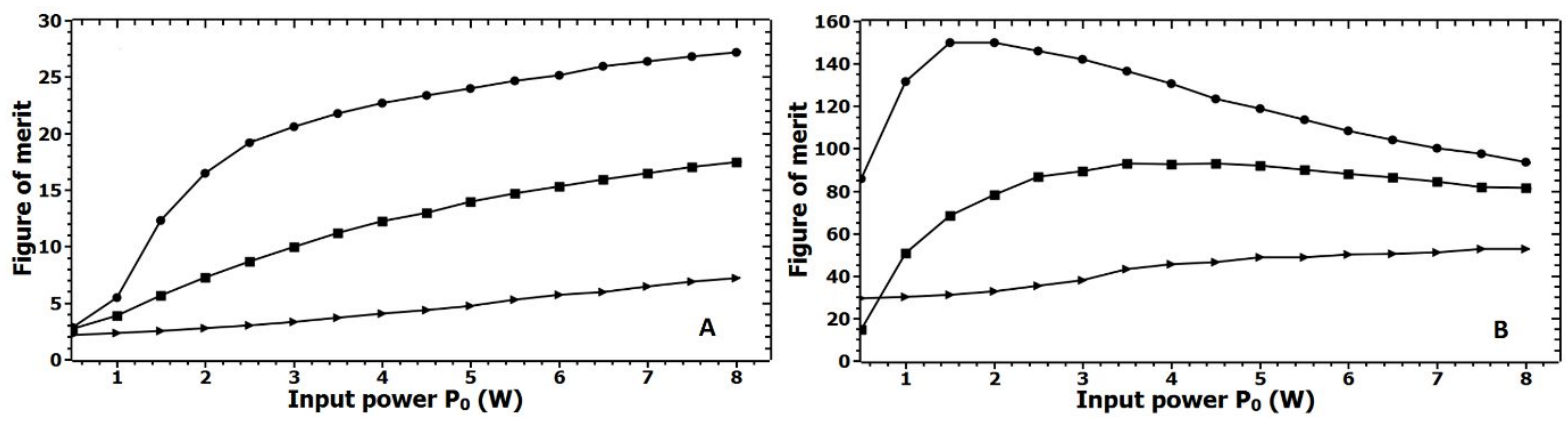

Fig. 7: Figure of merit of the 1. fibre (A) and the 2. fibre (B) depending on the input power $P_{0}$ and different values of the input laser separation: $L S=0.04 \mathrm{THz}$ (circles), $0.08 \mathrm{THz}$ (rectangles), and $0.16 \mathrm{THz}$ (triangles) 


\section{CONCLUSION}

We introduced a system consisting of three optical fibres which allows to generate high-quality ultra-short optical pulses with broad frequency-combs spectra from the two initial CW lasers. We numerically studied the dependence of the optimum lengths of the first and second fibre on the group-velocity dispersion and the nonlinear parameter of the first fibre and the initial laser frequency separation of the CW lasers. Since the widest possible spectral range of an optical frequency comb depends on the peak power and coincides with the minimal pulse width in the time domain, we introduced a figure of merit which represents the ratio between the peak power and the averaged power of the optical pulses. Moreover, we studied the dependence of the figure of merit again on the group-velocity dispersion and the nonlinear parameter of the first fibre and the initial laser frequency separation. A discussion of the noise evolution in the system was made.

\section{ACKNOWLEDGEMENTS}

We acknowledge the financial support of the German Federal Ministry of Education and Research (Grant 03ZAN11).

\section{REFERENCES}

[1] Griest, K., Whitmore, J. B., Wolfe, A. M., Prochaska, J. X., Howk, J. C., Marcy, G. W., "Wavelength accuracy of the Keck HIRES spectrograph and measuring changes in the fine structure constant", The Astrophysical Journal, 708:158$170(2010)$

[2] Jones, D. J., Diddams, S. A., Ranka, J. K., Stentz, A., Windeler, R. S., Hall, J. L., Cundiff, S. T. "Carrier-envelope phase control of femtosecond mode-locked lasers and direct optical frequency synthesis", Science, Vol. 288, No. 5466 (2000)

[3] Holzwarth, R., Udem, T., Hänsch, T. W., "Optical frequency synthesizer for precision spectroscopy", Physical Review Letters, Vol. 85, No. 11(2000)

[4] Cundiff, S. T., Yen, J., "Colloquium: femtosecond optical frequency combs", Reviews of Modern Physics, Vol. 75 (2003)

[5] Wilken, T., Lovis, C., Manescau, A., Steinmetz, T., Pasquini, L., Lo Curto, G., "High-precision calibration of spectrographs using laser frequency combs", Proc. Of SPIE, Vol. 7735 77350T-1 (2010)

[6] Steinmetz, T., WIlken T., Araujo-Hauck, A., Holzwarth, R., Hänsch, T. W., Pasquini, L., Manescau, A., D’Odorico, S., Murphy, M. T., Kentischer, T., Schmidt, W., Udem, T., "Laser frequency combs for astronomical observations", Science, Vol. 321, No. 5894 (2008)

[7] Murphy, M. T., Udem, T., Holzwarth, R., Sizmann, A., Pasquini, L., Araujo-Hauck, C., Dekker, H., D’Odorico, S., Fischer, M., Hänsch, T. W., Manescau, A., "High-precision wavelength calibration of astronomical spectrographs with laser frequency combs", Monthly Notices of the Royal Astronomical Society, Vol. 380, No. 2 (2007)

[8] Braje, D. A., Kirchner, M. S., Osterman, S., Fortier, T., Diddams, A., “Astronomical spectrograph calibration with broad-spectrum frequency combs", European Physical Journal D, Vol. 48, Issue 1 (2008)

[9] Loeb, A., "Direct measurement of cosmological parameters from the cosmic deceleration of extragalactic objects", Astrophysical Journal Letters, Vol. 499 (1998)

[10] Chavez Boggio, J. M., Rieznik, A. A., Zajnulina, M., Böhm, M., Bodenmüller, D., Wysmolek, M., Sayinc, H., Neumann, J., Kracht, D., Haynes, R., Roth, M. M., "Generation of an astronomical optical frequency comb in three fibre-based nonlinear stages”, Proc. SPIE 8434, Nonlinear Optics and Applications VI, 84340Y (2012)

[11] Li, Q., Kunz, J. N., Wai, P. K. A., "Cascaded higher-order soliton for non-adiabatic pulse compression”, Journal of Optical Society of America B, Vol. 27, No. 11 (2010) 
[12] Colman, P., Husko, C., Combrié, S., Sagnes, I., Wong, C. W., De Rossi, A., "Temporal solitons and pulse compression in photonic crystal waveguides", Nature Photonic, Vol. 4 (2010)

[13] Mollenauer, L. F., Stolen, R. H., Gordon, J. P., Tomlinson, W. J., „Extreme picosecond pulse narrowing by means of soliton effect in single-mode optical fibers“, Optics Letters, Vol. 8, No. 5 (1983)

[14] Hodel, W., Weber, H. P., "Decay of femtosecond higher-order solitons in an optical fiber induced by Raman selfpumping", Optics Letters, Vol. 12, No. 11 (1987)

[15] Porsezian, K., Nakkeeran, K., "Optical solitons in presence of Kerr dispersion and self-frequency shift”, Physical Review Letters, Vol. 76, No. 21 (1996)

[16] Cao, W., P. K. A. Wai, "Higher-order soliton compression with pedestal suppression in nonlinear optical loop mirrors constructed from dispersion decreasing fibers", Optics Communication 221 (2003)

[17] Voronin, A. A., Zheltikov, A. M., "Soliton-number analysis of soliton-effect pulse compression to single-cycle pulse width", Physical Review A 78, 063834 (2008) 